\title{
27
}

\section{Viability for differential inclusions in Banach spaces}

\author{
Ovidiu Cârjă \\ Facultatea de Matematică, Universitatea "Al.I.Cuza" \\ Bdul. Copou 11, 6600 Iaşi, România \\ Fax: 40-32-146330. E-mail: carja@uaic.ro
}

\begin{abstract}
The aim of this paper is to give a sufficient condition in order that a subset of a Banach space be a viability domain for a semilinear differential inclusion.
\end{abstract}

\section{Keywords}

Viability, semilinear differential inclusion, tangency condition

\section{INTRODUCTION AND MAIN RESULT}

Let $X$ be a Banach space, $D$ a nonempty subset of $X, F: D \rightarrow X$ a multifunction with nonempty values and $A$ the infinitesimal generator of a $c_{0}$ semigroup $S(t)$ on $X$. Consider the differential inclusion

$x^{\prime}(t) \in A x(t)+F(x(t))$.

By a viable solution to (1) we mean a continuous function $x:[0, \sigma] \rightarrow X$ which satisfies $x(t) \in D$ for all $t \in[0, \sigma]$ and there exists a strongly measurable function $f$ from $[0, \sigma]$ into $X$ with $f(s) \in F(x(s))$ a.e., such that

$x(t)=S(t) x(0)+\int_{0}^{t} S(t-s) f(s) d s, \forall t \in[0, \sigma]$.

The set $D$ is called a viability domain for the differential inclusion (1) if for every $x_{0} \in D$ there exists a viable solution to (1) with $x(0)=x_{0}$. The viability problem is to give conditions in order that the set $D$ be a viability domain for (1).

In the finite dimensional case (where $A=0$, hence $S(t)=I$ ), the main viability result is due to Gautier (1973) and Haddad (1981). See also Aubin and Cellina (1984), Cârjă and Ursescu (1993). It asserts that if $F$ is upper semicontinuous with compact convex values and $D$ is locally closed, then $D$ is a viability domain for (1) if and only if the tangency condition

$F(x) \cap T_{D}(x) \neq 0, \quad \forall x \in D$, 
is satisfied. Here the tangency concept $T_{D}$ is that of Bouligand and Severi: $u \in T_{D}(x) \Longleftrightarrow$ $\liminf _{t \downarrow 0} \frac{1}{t} d(x+t y, D)=0$. where $d(a, B)=\inf _{b \in B}\|a-b\|$.

The viability result mentioned above has served as a main tool in a general study of the characteristics method for a first order partial differential equation (see Cârjă and Ursescu (1993)). In particular, this leads to a study of the Hamilton-Jacobi-Bellman equations from the point of view of contingent solutions (see Cârjă (1996)). In infinite dimensions, for differential inclusion of type (1) we mention two important results. The first one is due to Pavel and Vrabie (1979) (see also Pavel (1984), Chapter 5), where $S(t)$ is compact, $F$ is locally bounded, demiclosed with closed convex values, and $D$ is locally closed. They got viable solutions to (1) for every $x_{0} \in D$ from the tangency condition

$\lim _{t ! 0} \frac{1}{t} d(S(t) x+t y, D)=0, \quad \forall x \in D, \forall y \in F(x)$.

The second result is due to ShiShuzhong (1989), where $S(t)$ is a compact differentiable semigroup, $D$ is compact and $F$ is upper semicontinuous with compact convex values. He proved that under these conditions $D$ is a viability domain for (1) if and only if the tangency condition

$F(x) \cap T_{D}^{S}(x) \neq 0, \quad \forall x \in D$

is satisfied, where the tangency concept $T_{D}^{S}$ is defined by

$u \in T_{D}^{S}(x) \Longleftrightarrow \liminf _{t \downarrow 0} \frac{1}{t} d(S(t) x+t u, D)=0$.

In many situations, especially in the study of the Bellman equation associated with control problems, the hypotheses in ShiShuzhong (1989) that $D$ is compact and $F$ has compact values are too strong. A very simple example is the linear control system $x^{\prime}(t) \in$ $A x(t)+B U$, where $U$ is a bounded closed convex set and $B$ is a linear continuous operator.

On the other hand, the tangency condition in Pavel and Vrabie (1979) is also too strong in comparison to that of the finite dimensional case.

The aim of this note is to show that the tangency condition (3) in Pavel and Vrabie (1979) can be replaced by the weaker condition (4). The price to be paid is to strengthen the conditions on $F$. Namely, in addition to the other hypotheses we assume that $F$ is "strongly-weakly" lower semicontinuous. Precisely, we have

Theorem 1 Let $X$ be a reflexive Banach space and $D$ a locally closed subset of $X$. Assume: $S(t)$ is compact for $t>0 ; F$ is locally bounded, demiclosed and lower semicontinuous with closed convex values; the tangency condition (4) holds true. Then for every $x_{0} \in D$ there exists a viable solution to the differential inclusion (1).

Let us define the notions lower semicontinuous and demiclosed.

We say that the multifunction $F: D \rightarrow X$ is lower semicontinuous in $x \in D$ if $\forall y \in F(x), \forall\left(x_{n}\right) \in D, x_{n} \rightarrow x$, there exists $y_{n} \in F\left(x_{n}\right)$ such that $y_{n} \rightarrow y$.

We say that the multifunction $F$ is demiclosed if the hypotheses: $y_{n} \in F\left(x_{n}\right)$, with $x_{n} \in D, x_{n} \rightarrow x$ and $y_{n} \rightarrow y$ imply $x \in D$ and $y \in F(x)$. 
Mention that we use the same idea of proof as in Pavel and Vrabie (1979) and Pavel (1984), therefore some steps will only be sketched. Finally, note that in Pavel and Vrabie (1979) and Pavel (1984) both $D$ and $F$ depend on $t$ but we consider the autonomous case here for simplicity only.

\section{PROOF OF THE MAIN RESULT}

As we remarked earlier, we follow the same line as in Pavel and Vrabie (1979) and Pavel (1984). By the nonexpansivity of the distance function it follows that the tangency concept $T_{D}^{S}$ defined in (5) is equivalent to the following one:

$u \in T_{D}^{S}(x) \Longleftrightarrow \liminf _{h \downarrow 0}(1 / h) d\left(S(h) x+\int_{0}^{h} S(h-s) u d s, D\right)=0$.

Since $F$ is locally bounded and $D$ is locally closed, for $x_{0} \in D$ there are constants $M>0, r>0$ such that $B\left(x_{0}, r\right) \cap D$ is closed and

$\|f(y)\| \leq M, \forall y \in B\left(x_{0}, r\right) \cap D, \forall f(y) \in F(y)$.

Here $B\left(x_{0}, r\right)$ is the closed ball of center $x_{0}$ and radius $r$.

Let $T$ be sufficiently small such that $\max _{0 \leq t \leq T}\left\|S(t) x_{0}-x_{0}\right\|+T(M+1) K \leq r$

where $K=C \exp (\omega T)$ and $C \geq 1, \omega \geq 0$ are such that $\|S(t)\| \leq C \exp (\omega t)$ for $t \geq 0$.

The following lemma concerns the construction of an approximate solution to (1) on $[0, T)$. This is the main step of the proof of Theorem 1 .

Lemma 1 Suppose that the hypotheses of Theorem 1 hold. Let $x_{0} \in D$ and choose $r, T, M$ as in (7) and (8). Then there is an $(1 / n)$-approximate solution $x_{n}$ to (1) on $[0, T)$ in the following sense: For each positive integer $n$, there is an infinite partition $\left\{t_{i}^{n}\right\}_{i \geq 0}$ of $[0, T]$ with the following properties:

(P1) $t_{0}^{n}=0, t_{i+1}^{n}-t_{i}^{n}:=d_{i}^{n} \in\left(0, \frac{1}{n}\right], \quad \lim _{i \rightarrow \infty} t_{i}^{n}=T$;

(P2) $x_{n}(0)=x_{0}, \quad x_{n}\left(t_{i}^{n}\right):=x_{i}^{n} \in B\left(x_{0}, r\right) \cap D$;

(P3) $x_{n}(t)=S\left(t-t_{i}^{n}\right) x_{i}^{n}+\int_{t_{i}^{n}}^{t} S(t-s) f\left(x_{i}^{n}\right) d s+\left(t-t_{i}^{n}\right) p_{i}^{n} \in B\left(x_{0}, r\right)$ for $t \in\left[t_{i}^{n}, t_{i+1}^{n}\right]$ with $\left\|p_{i}^{n}\right\| \leq \frac{1}{n}$, where $f\left(x_{i}^{n}\right) \in F\left(x_{i}^{n}\right)$.

Proof. To simplify notation, suppress $n$ as a superscript for $t_{i}, d_{i}, x_{i}$ and $p_{i}$. The construction is by induction. Set $t_{0}=0, x_{n}(0)=x_{0}$, and assume that $x_{n}$ is constructed on $\left[0, t_{i}\right]$. If $t_{i}=T$, set $t_{i+1}=t_{i}$. Consider now the case $t_{i}<T$. Taking into account that $x_{i} \in D$, by (4) and (6) there exists $f\left(x_{i}\right) \in F\left(x_{i}\right)$ such that

$\liminf _{h \backslash 0}(1 / h) d\left(S(h) x_{i}+\int_{0}^{h} S(h-s) f\left(x_{i}\right) d s, D\right)=0$.

This implies the existence of $h_{i} \in(0,1 / n]$ with $t_{i}+h_{i} \leq T$ such that

$d\left(S\left(h_{i}\right) x_{i}+\int_{0}^{h_{i}} S\left(h_{i}-s\right) f\left(x_{i}\right) d s, D\right) \leq \frac{h_{i}}{2 n}$. 
We can thus define

$\delta_{i}=\sup \left\{h \in\left(0, \frac{1}{n}\right] ; t_{i}+h \leq T, \exists f\left(x_{i}\right) \in F\left(x_{i}\right)\right.$,

$\left.d\left(S(h) x_{i}+\int_{0}^{h} S(h-s) f\left(x_{i}\right) d s, D\right) \leq \frac{h}{2 n}\right\}$.

Therefore, there exist $d_{i} \in\left(\frac{\delta_{i}}{2}, \delta_{i}\right], 0<d_{i} \leq \frac{1}{n}, t_{i}+d_{i} \leq T$ and $f\left(x_{i}\right) \in F\left(x_{i}\right)$ such that $d\left(S\left(d_{i}\right) x_{i}+\int_{0}^{d_{i}} S\left(d_{i}-s\right) f\left(x_{i}\right) d s, D\right)<\frac{d_{i}}{2 n}$.

Set $t_{i+1}:=t_{i}+d_{i}$. By (10), there exists $x_{i+1} \in D$ such that

$\left\|S\left(d_{i}\right) x_{i}+\int_{0}^{d_{i}} S\left(d_{i}-s\right) f\left(x_{i}\right) d s-x_{i+1}\right\| \leq \frac{d_{i}}{n}$

i.e.,

$x_{i+1}=S\left(t_{i+1}-t_{i}\right) x_{i}+\int_{t_{i}}^{t_{i+1}} S\left(t_{i+1}-s\right) f\left(x_{i}\right) d s+\left(t_{i+1}-t_{i}\right) p_{i}, \quad w i t h\left\|p_{i}\right\| \leq \frac{1}{n}$.

Define $x_{n}$ on $\left[t_{i}, t_{i+1}\right]$ as indicated in (P3). It is easy to see that

$x_{n}(t)=S(t) x_{0}+\int_{0}^{t} S(t-s) f_{n}(s) d s+g_{n}(t), \quad t \in\left[0, t_{i+1}\right]$

where

$f_{n}(s)=f\left(x_{i}\right), \quad s \in\left[t_{j}, t_{j+1}\right], \quad j=0, \cdots, i$

and

$$
\begin{aligned}
& g_{n}(t)=\sum_{k=0}^{j-1}\left(t_{k+1}-t_{k}\right) S\left(t-t_{k+1}\right) p_{k}+\left(t-t_{j}\right) p_{j}, \quad t \in\left[t_{j}, t_{j+1}\right], \quad j=1, \cdots, i, \\
& g_{n}(t)=t p_{0}, \quad t \in\left[0, t_{1}\right] .
\end{aligned}
$$

By induction hypothesis, $x_{n}(t) \in B\left(x_{0}, r\right)$ for $t \in\left[0, t_{i}\right]$. We have to prove that $x_{n}(t) \in$ $B\left(x_{0}, r\right)$ for $t \in\left[t_{i}, t_{i+1}\right]$. Indeed, for $t \in\left[t_{i}, t_{i+1}\right]$,

$$
\left\|x_{n}(t)-x_{0}\right\| \leq\left\|S(t) x_{0}-x_{o}\right\|+T M K+\frac{T K}{n} \leq r
$$

because of (7), (8) and the fact that

$\left\|g_{n}(t)\right\| \leq \frac{K t}{n}$.

Therefore, if $t^{\star}:=\lim _{i \rightarrow \infty} t_{i}$, we can define $x_{n}(t)$ by (11) for every $t \in\left[0, t^{\star}\right)$ where $f_{n}$ is given by (12) for every $j \in N$ and $g_{n}$ is given by (13) for every $j \in N$. As results from Pavel (1984), $\left(x_{i}\right)$ is a Cauchy sequence, and let $x^{\star}$ be its limit. Clearly, $x^{\star} \in B\left(x_{0}, r\right) \cap D$.

We prove now that $t^{\star}=T$. It is this step where we use the fact that $F$ is lower semicontinuous instead of the condition (3). suppose to the contrary that $t^{\star}<T$. By (2), there exist $h^{\star} \in\left(0, \frac{1}{n}\right]$ with $h^{\star}+t^{\star}<T$ and $f^{\star} \in F\left(x^{\star}\right)$ such that

$$
d\left(S\left(h^{\star}\right) x^{\star}+\int_{0}^{h^{\star}} S\left(h^{\star}-s\right) f^{\star} d s, D\right) \leq \frac{h^{\star}}{4 n} .
$$


Since $d_{i} \rightarrow 0, \delta_{i} \rightarrow 0$ hence there exists $i_{0}$ such that for $i \geq i_{0}, h^{\star}>\delta_{i}$. Because $F$ is lower semicontinuous, there exists $y_{i} \in F\left(x_{i}\right)$ such that $y_{i} \rightarrow f^{\star}$. Since $t_{i} \leq t^{\star}$, we have $h^{\star}+t_{i}<T$. Along with $h^{\star}>\delta_{i}$, the very definition of $\delta_{i}$ implies

$$
d\left(S\left(h^{\star}\right) x_{i}+\int_{0}^{h^{\star}} S\left(h^{\star}-s\right) y_{i} d s, D\right)>\frac{h^{\star}}{2 n}, \quad i \geq i_{0}
$$

On the other hand by a standard argument,

$\int_{0}^{h^{\star}} S\left(h^{\star}-s\right) y_{i} d s \rightarrow \int_{0}^{h^{\star}} S\left(h^{\star}-s\right) f^{\star} d s$

Passing to limit in (15) we get a contradiction with (14). Therefore $t^{\star}=T$ as claimed. This ends the proof of Lemma 1.

Proof of Theorem 1. It follows as in Pavel (1984), p.190. One proves that the sequence $\left(x_{n}\right)$ constructed in Lemma 1 has a convergent subsequence in $C([0, T] ; X)$ to a solution of (1). The Ascoli-Arzela theorem and a result of Kato are the main tools.

\section{REFERENCES}

Aubin, J. P. and Cellina, A. (1984) Differential Inclusions, Springer-Verlag, Berlin.

Cârjă, O. (1996) Lower semicontinuous solutions for a class of Hamilton-Jacobi-Bellman equations, Journal of Optimization Theory and Applications, 89.

Cârjă, O. and Ursescu, C. (1993) The characteristics method for a first order partial differential equation, Analele Ştiinţifice ale Universităţii Al.I.Cuza Iaşi, 39, pp. 367396.

Cârjă, O. and Ursescu, C. (1994) Viscosity solutions and partial differential inequations, in Evolutions Equations, Control Theory and Biomathematics (eds. P. Clement and G. Lumer), Marcel Dekker, New York, pp. 39-44.

Gautier, S. (1973) Equations différentielles multivoque sur un fermè , Publications de l'Université de Pau, pp. 1-5.

Haddad, G. (1981) Monotone trajectories of differential inclusions and functional differential inclusions with memory, Israel Journal of Mathematics, 39, pp. 83-100.

Pavel, N. H. and Vrabie, I. I. (1979) Semi-linear evolution equations with multivalued right hand side in Banach spaces, Analele Ştiinţ̧ifice ale Universităţii Al.I.Cuza Iaşi, 25, pp. 137-157.

Pavel, N. H. (1984) Differential Equations, Flow Invariance and Applications, Research Notes in Mathematics, 113, Pitman, London.

ShiShuzhong, (1989) Viability theorems for a class of differential-operator inclusions, Journal of Differential Equations, 79, pp. 232-257. 